\title{
Proton driver for IsoDAR
}

Joseph Smolsky* for the IsoDAR collaboration

Massachusetts Institute of Technology

E-mail: smolsky@mit.edu

The Isotope Decay-At-Rest (IsoDAR) experiment is a short baseline neutrino experiment designed to measure neutrino oscillations by placing a high flux electron anti-neutrino source near a kiloton scale scintillator detector. A high current proton beam will be used to produce the large number of electron anti-neutrinos needed. Reaching the design goal of $10 \mathrm{~mA}$ of $60 \mathrm{MeV}$ protons on target requires advancements in accelerator technology. This can be achieved using a high intensity $\mathrm{H}_{2}^{+}$ion source followed by a radio-frequency quadrupole and spiral-inflector to axially inject ions into a compact cyclotron. Presented here are the results of our latest studies of this injection system.

The 20th International Workshop on Neutrinos (NuFact2018)

12-18 August 2018

Blacksburg, Virginia

${ }^{*}$ Speaker. 


\section{Introduction: IsoDAR}

The Isotope Decay-At-Rest (IsoDAR) experiment is primarily a search for sterile neutrinos: non-weakly interacting neutrino flavor(s) proposed to explain anomalies seen at short baseline and reactor neutrino experiments. It will also be a search for non-standard interactions through $\bar{v}_{e}-e$ elastic scattering events and precision measurement of electroweak couplings and mixing[1]. Other uses of the IsoDAR cyclotron include measuring $\delta_{c p}$ in the DAE $\delta$ ALUS experiment[2] and medical isotope production[3].

The physics comes from a high intensity proton beam used to produce electron anti-neutrinos near a kiloton scale detector. $\mathrm{H}_{2}^{+}$ions will be axially injected into a compact cyclotron, producing $10 \mathrm{~mA}$ of $60 \mathrm{MeV}$ protons. The protons impinge on a ${ }^{9} \mathrm{Be}$ target producing neutrons that are captured by a surrounding ${ }^{7} \mathrm{Li}$ sleeve, producing ${ }^{8} \mathrm{Li}$. The ${ }^{8} \mathrm{Li} \beta$-decays at rest and serves as the $\bar{v}_{e}$ source. The source is placed 12-16 m from a kiloton scale detector. Pairing with the KamLAND detector would result in over 800,000 inverse $\beta$-decay events and approximately 2,600 $\bar{v}_{e}-e$ elastic scattering events in five years[1]. The focus herein is ion production and acceleration.

\section{Injection System and Cyclotron}

Commercial cyclotrons typically accelerate $\mathrm{H}^{-}$and output currents around $1 \mathrm{~mA}$. To produce $10 \mathrm{~mA}$ of medium energy protons requires new technology. This is being developed as part of the Radio Frequency Quadrupole-Direct Injection Project (RFQ-DIP) and is discussed in detail in the article High intensity cyclotrons for neutrino physics [4]. The key changes are accelerating $\mathrm{H}_{2}^{+}$to reduce space-charge effects in the cyclotron and axial injection using an RFQ to increase injection efficiency.

The $\mathrm{H}_{2}^{+}$ion source is currently being commissioned at MIT. A heated tungsten filament ionizes hydrogen molecules. A mutli-cusp field created by permanent magnets contains the plasma in a small chamber. A small hole opposite the filament allows ions to drift into the extraction system. The extraction system focuses the ions and accelerates to $15 \mathrm{keV}$. The design goal is $50 \mathrm{~mA} \mathrm{~cm}{ }^{-2}$ of $\mathrm{H}_{2}^{+}$. Preliminary measurements have been up to $37 \mathrm{~mA} \mathrm{~cm}^{-2}$ of total current. However, species analysis has yet to be done at this time and is expected to take place early next year. A new extraction system is also being designed that will lower beam emittance and allow for minor steering into the RFQ through slits in the lenses.

After extraction, ions will be simultaneously separated, bunched, and slightly accelerated by the RFQ. The purpose of the RFQ is to increase injection efficiency into the cyclotron by providing bunches in phase with the RF of the cyclotron; the acceleration by the RFQ will only be to $70 \mathrm{keV}$. A 4-vane, split-coaxial design is currently being modeled and simulated. A key element of the RFQ design is a re-bunching cell placed at the end of the RFQ. This allows the bunches to grow slightly before the final focusing element, putting the focal point of the RFQ in the beginning of the cyclotron RF-cavity. Simulations predict over $97 \%$ transmission efficiency from the RFQ[4].

The ion source and RFQ are axially aligned through the cyclotron yoke. To make the $90^{\circ}$ bend into the cyclotron mid-plane, a spiral-inflector will be utilized. Two copper electrodes, $1 \mathrm{~cm}$ wide and $1.8 \mathrm{~cm}$ apart at $\pm 12 \mathrm{kV}$, are formed in a spiral to steer $\mathrm{H}_{2}^{+}$ions into the central region of the cyclotron. Significant beam loss is expected as ions go through the spiral inflector and central 
region, but the beam current will still be sufficient given the high current possible from the ion source and RFQ.

Design of the central region is currently underway by AIMA Developpement using output from the spiral inflector simulations. The central region will contain collimators to scrape halo particles coming from the spiral inflector. A feature of the yoke is vanadium permendur inserts on the pole tips for vertical focusing. The RFQ-DIP includes testing of the central region design in a $1 \mathrm{MeV}$ cyclotron. The same design will then implemented in the $60 \mathrm{MeV}$ cyclotron for IsoDAR.

After the central region is a fairly typical 4th-harmonic cyclotron, up to the point of extraction. The intensity of the beam would cause the electrostatic septum used in extraction to become highly activated. To prevent this, a stripper-foil removes electrons from halo particles causing them to follow a different trajectory out of the cyclotron. This controlled beam loss of a few $\mu$ A protects the septum and can also be used to produce medical isotopes. The remaining $\mathrm{H}_{2}^{+}$can be safely extracted once the halo has been removed. A second stripper-foil outside of the cyclotron will be used so the beam can be transported as protons to the target. This can be done using well established methods for medium energy beam transport.

\section{Beyond IsoDAR: Medical Isotopes and DAE $\delta$ ALUS}

Two exciting possibilities for IsoDAR beyond the sterile neutrino search are medical isotope production and the DAE $\delta$ ALUS experiment. Details of isotope production possibilities are discussed in the article Isotope Production from the IsoDAR Cyclotron and include exceeding current world production rates of some $\alpha$-emitters and $\beta$-emitters used for imaging and treatment[3]. Of more interest to particle physicists is the possibility of using IsoDAR cyclotrons as an injection systems for the DAE $\delta$ ALUS cyclotrons.

For DAE $\delta$ ALUS, the $60 \mathrm{MeV}$ protons get injected into $800 \mathrm{MeV}$ segmented cyclotrons. The $800 \mathrm{MeV}$ protons are used to produce $\bar{v}_{\mu}$ through a stopped pion beam. Three such cyclotrons, at baselines ranging 1-15 km away from a 20-50 kiloton detector, provide a means to study CP-violation in the neutrino sector. More information on this experiment is available on the DAE $\delta$ ALUS-IsoDAR website [2].

\section{References}

[1] J.R. Alonso1 and K. Nakamura for the IsoDAR Collaboration, A Conceptual Design Report for the Conventional Facilities, (2017) [arXiv:1710.09325 [physics.ins-det]].

[2] About IsoDAR and DAESALUS, (2018) [https://www.nevis.columbia.edu/daedalus/].

[3] Jose R Alonso, Janet Conrad, Loyd Hoyt Waites, Isotope Production from the IsoDAR Cyclotron, (2018) [arXiv:1807.06627 [physics.acc-ph]].

[4] Daniel Winklehner, Jungbae Bahng, Luciano Calabretta, Alessandra Calanna, Alok Chakrabarti, Janet Conrad, Grazia D’Agostino, Siddharta Dechoudhury, Vaishali Naik, Loyd Waites, Philip Weigel, High intensity cyclotrons for neutrino physics, (2018) [arXiv:1807.03759v2 [physics.acc-ph]]. 